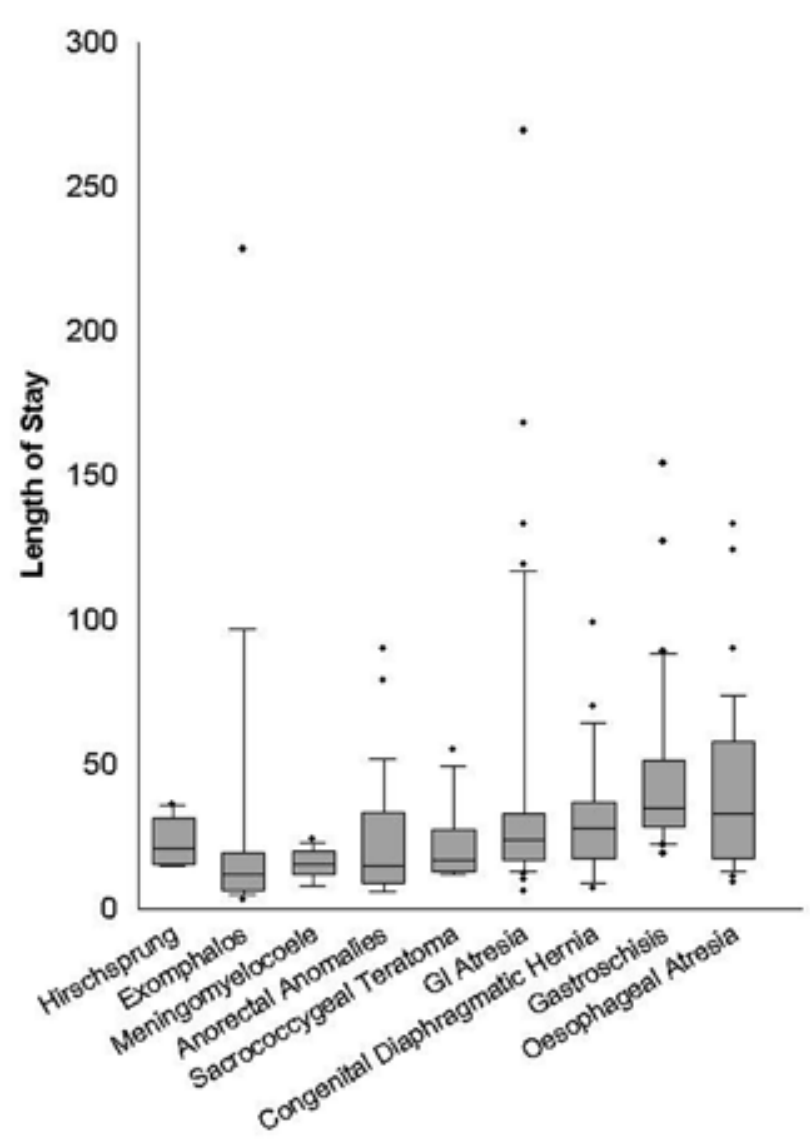

Abstract 818 Graph 1 Box and Whisker Plots of LOS

Conclusion Our data for length of stay LOS for following neonatal surgery compare favourably to historical data. Our data show a wide variation in lengths of stay; we believe that median LOS with ranges will enable us to give more detailed information to families at diagnosis.

These data allow more detailed planning of resource allocation when planning admissions of these often complex babies.

\section{THE EFFECT OF NEONATAL UNIT CARE LEVEL AND VOLUME ON MORTALITY, DISCHARGE, AND TRANSFER: EVIDENCE FROM ENGLISH HOSPITALS}

doi:10.1136/archdischild-2012-302724.0819

'S Watson, 'S Petrou, ${ }^{2} \mathrm{~W}$ Arulampalam, ${ }^{3} \mathrm{~N}$ Modi. 'Warwick Medical School; ${ }^{2}$ Department of Economics, University of Warwick, Coventry; ${ }^{3}$ Academic Neonatal Medicine, Imperial College London, London, UK

Background s/aims: In the US a low volume of admissions is associated with higher mortality in very low birth weight (VLBW) infants leading to recommendations for centralisation of neonatal services. We examined the likelihood of mortality, discharge, and transfer for VLBW and/or $<33+0$ week gestation babies in relation to volume of admissions and care level in England.

Methods Competing risks regression, allowing clustering at the unit level, was used with data from the first episode of care. Units were classified by level of care and tertile of volume.

Results Following case-mix adjustment, relative to highest volume level three (highest intensity) units, top-tertile level one was associated with reduced risk of mortality (OR:0.05; CI:0.01-0.35; $\mathrm{p}=0.002$ ). Level one and lower tertile level two units were associated with increased probability of discharge (eg top-tertile level one,
OR:1.91; CI:1.43-2.55; p<0.001). Level one and level two units were less likely to transfer (eg top-tertile level one, OR:0.49; CI:0.33-0.73; $\mathrm{p}<0.001$ ). These effects became statistically insignificant once 'high risk' babies (with congenital abnormalities, requiring surgery, and born $<29$ weeks gestation) were removed.

Conclusions In this UK study we show reduced mortality in level one relative to level three units, and that this difference is explained by a less severe case-mix in lower level units. The majority of care for high risk babies in England is appropriately delivered by highlevel units. In the US the case-mix of high- and low-level units is similar. We suggest a network based approach achieves the benefits of centralisation without the disadvantages.

\section{LATE PRETERM DELIVERIES AND NEONATAL OUTCOMES IN MULTICENTRE FRENCH REGIONAL PERINATAL NETWORK}

doi:10.1136/archdischild-2012-302724.0820

${ }^{1} \mathrm{M}$ Le Duin, ${ }^{2} \mathrm{C}$ Tripon, ${ }^{3} \mathrm{~K}$ Husseini, ${ }^{4} \mathrm{~J}$ Cuzzi, ${ }^{4} \mathrm{D}$ Lapeyre, ${ }^{4} \mathrm{Y}$ Wardi, ${ }^{4} \mathrm{~F}$ Hay-Findler. 'Obstetrics; ${ }^{2}$ Neonatology; ${ }^{3}$ Pediatric Intensive Care Unit; ${ }^{4}$ University Hospital of Poitiers, Poitiers, France

Objective The objective of the study was to present the neonatal outcomes for late preterm birth by gestational age at 33.34 and 35 weeks in a multicentric neonatal network.

Study Design This was a retrospective analysis of neonatal outcomes of late preterm infants (33, 34 and 35 weeks groups) in 5 neonatal care units with one University Hospital Level 3 NICU in one regional perinatal network during 2010. Data were analyzed using Chi(2), Stuent's Test, and one way ANOVA test.

Results During 2010, the rate of late preterm birth 299/8718 births: $3.42 \pm 0.94 \%$ without significant difference between the 5 centres. Rates were $0.62 \%, 1.35 \%$ and $1.46 \%$ respectively at 33,34 and 35 wks. There was $19 \%$ of twin pregnancy. Vaginal birth rate $(57.6 \%)$ was not significantly different between groups. Increased gestational age was associated with decreased antenatal steroid use $(80.8 \%, 47.4 \%$ and $20.6 \%$ respectively; $\mathrm{p}=0.001)$. Neonatal mortality was $2 / 299(0.68 \%)$. Postnatal transport was low $(4.3 \%$ at $33,4.1$ at 34 and 5.6 at 35$)$. Respiratory distress decreased (27.8\% at 33 vs $8.5 \%$ at 34 and $7.9 \%$ at $35 ; \mathrm{p}=0.001$ ) without significant difference in the use of surfactant. Feeding problems decreased $(20.4 \%$ at 33 vs $5.5 \%$ at $35 ; \mathrm{p}=0.01)$. There was no significant difference in gestational age at hospital discharge between groups $(37.07 \pm 1.3$ at 33 , $36.9 \pm 1.2$ at 34 and $37.3 \pm 1.1$ ).

Conclusion Compared to recent studies the rate of late preterm delivery in our region was similar. Low rate of postnatal transport showed good organization of the regional perinatal network.

\section{AN AUDIT OF THE USE OF PICC LINES IN PRETERM INFANTS ( $<33$ WEEKS) IN A TERTIARY NEONATAL INTENSIVE CARE UNIT}

doi:10.1136/archdischild-2012-302724.0821

${ }^{1}$ A Nosherwan, 1 I Gill, 'H Walsh, ${ }^{2} \mathrm{~S}$ Knowles, 'A Twomey. ${ }^{1}$ Neonatology; ${ }^{2}$ Microbiology, National Maternity Hospital, Dublin, Ireland

Aim To study the frequency of use, indications for placement and complications associated with PICC line placement in a single tertiary NICU.

Method A prospective review of all the PICC lines inserted over ten-month period. Data was obtained from clinical notes, radiology reports and laboratory results.

Results A total of 71 lines were placed in 53 infants $<33$ weeks. $43 \%(53 / 131)$ infants < 33 weeks admitted had PICC line placed. The mean gestational age was $27.7 \pm 2.3$ weeks and mean birth weight was $1030 \pm 332 \mathrm{~g}$. The indications for insertion was administration of Parenteral nutrition (86\%), Inotropes (11\%), and antibiotics $(3 \%)$. The mean age at insertion was $7 \pm 6$ days and the average 
duration of placement was $6 \pm 5$ days. All the lines were inserted with maximum sterile barrier and $86 \%$ were accessed once per day under strict sterile protocol. 35\% lines were correctly placed, $64 \%$ required manipulation and post manipulation catheter tip was confirmed in $64 \%$ cases. $58 \%$ of the lines completed treatment. Line occlusion was the most common complication (17\%), which significantly reduced the duration of line placement by 3.3 days $(p=0.02)$. The infection rate was 13 per 1000 catheter days.

Conclusion There is a high rate of PICC associated complications with occlusion of lines accounting for most of these. Our NICU is reviewing whether thrombolytic agents should be considered routinely for line occlusion. Attention has been directed to ensure that line position is reconfirmed by X-ray after manipulation. Our infection rate still remains high when compared to rates quoted internationally.

\section{DESCRIBING THE USE OF NEONATAL THERAPEUTIC INTERVENTION SCORING SYSTEM IN A UNIVERSITY HOSPITAL IN BRAZIL - A PILOT STUDY}

doi:10.1136/archdischild-2012-302724.0822

${ }^{1} \mathrm{CG}$ Carvalho, ${ }^{2} \mathrm{M}$ Ribeiro, ${ }^{2} \mathrm{BC}$ Benincasa, ${ }^{1} \mathrm{RS}$ Procianoy, ${ }^{1} \mathrm{RC}$ Silveira. ${ }^{1} \mathrm{HCPA}$ - UFRGS; ${ }^{2}$ UFRGS, Porto Alegre, Brazil

Background and Aims The Neonatal Therapeutic Intervention Scoring System (NTISS) is an index of intensity of use of technologies with significant association to mortality risk. We intended to analyze this score use in the NICU of a university hospital, correlating with mortality.

Method It was a prospective cohort including 129 newborns admitted to NICU during a 6-week-period. Patients were followed for up to 31 days, with daily calculation of NTISS. Demographic data were all obtained by review of medical records, under informed consent. For statistical analysis It was used the $\mathrm{x}^{2}$ and Mann-Whitney tests.

Results The most frequent cause of hospitalization was neonatal jaundice (35\%), followed by early respiratory dysfunction (16\%) and prematurity (13\%), the mean hospital stay was 10 days. The median NTISS was 6 on the first day and remained stable in the next days. Only 4 patients died during the study - with a first day NTISS median of 28 vs $6(p=0.002)$. This behavior continued until the seventh day and SNAPPEII score also showed this kind of tendency. Patients with extreme prematurity, congenital malformation or early respiratory dysfunction that remained hospitalized until 31 days exhibited NTISS values greater than the median until the 27 th day of admission.

Conclusion The NTISS, at least in the first week of hospitalization, was higher in patients who die, as a predictor of mortality in this sample. The NTISS remained high under conditions known to be severe for a long period of hospitalization, which may affect heath costs.

\section{CLINICAL PROFILE OF PATIENTS AND PREDICTIVE VALUE OF PIM 2 SCORE AT PAEDIATRIC INTENSIVE CARE UNIT IN WESTERN INDIA}

doi:10.1136/archdischild-2012-302724.0823

'V Shukla, 1,2SM Nimbalkar, ${ }^{2} \mathrm{JD}$ Ganjiwale, ${ }^{2} \mathrm{AG}$ Phatak. 'Department of Pediatrics, Pramukhswami Medical College, ${ }^{2}$ Central Research Services, Charutar Arogya Mandal, Anand, India

Background and Aims PIM2 score gives the risk score for specific clinical disease condition. We studied the profile of patients admitted in PICU and validated PIM2 score in our tertiary care setting. Methods Detailed evaluation of patients admitted to the PICU between January 2010 and December 2011 was done for presenting signs and symptoms, management by trauma team and pediatric team and the appropriateness of the intereventions were recorded till the child had spent 24 hours in the hospital. Outcomes were death, discharge and DAMA. The PIM2 scoring was done at the time of admission. Data was analyzed by Logistic regression and ROC curve analysis.

Results Of 742 consecutive admissions (295 females and 447 males, $39.08 \%$ infants), $35.84 \%$ patients were mechanically ventilated and mortality was $7 \%$. Significant anemia (27.2\%), pneumonia $(19.5 \%)$, meningitis/encephalitis $(17.1 \%)$, septicemia and septicemic shock $(29.5 \%)$ were seen. $25.6 \%$ patients admitted in PICU took discharge against medical advice due to unaffordable medical care. Sensitivity of PIM2 score was $65.7 \%$, and specificity was $70.6 \%$ at cut-off point of 1.9 . Predictive capability as assessed by calculating the area under ROC curve was 0.724 (95\% CI 0.69 to 0.76). Logistic Regression analysis revealed that age, shock, Protein energy malnutrition, multiple organ dysfunction syndrome, meningitis/encephalitis, adherence to guidelines, ventilatory support requirement are significant predictors of Mortality.

Conclusions Infectious diseases were the most common cause of PICU admissions and mortality. PIM2 scoring did not correlate well with outcome suggesting need of recalibration. Following published emergency guidelines was associated with significantly better outcome.

\section{PHYSIOTHERAPY TECHNIOUES FOR PLEURAL EFFUSIONS IN A PEDIATRIC INTENSIVE CARE UNIT (PICU): WHICH TECHNIQUE IS THE MOST EFFICIENT?}

doi:10.1136/archdischild-2012-302724.0824

S Cooreman, V van Gorp, N Najafi, L Huyghens, S Hachimi-Idrissi. Critical Care Department, UZ Brussel, Brussels, Belgium

Aims To compare different chest physiotherapy techniques in pediatric patient with infectious pleural effusion and thorax drain admitted in PICU

Methods We conducted a monocentric, randomized, assessorblind trial. The patients were randomized in three groups: those who have received intrapulmonary percussive ventilation (IPV), those who have received autogene drainage $(\mathrm{AD})$ and were compared to the control group (CG) which no physiotherapy was administered. Only physiotherapists were aware of the allocation group of the patients.

Results 24 patients were included (IPV: 7, AD:8, CG: 9). All patients had a infectious pleural effusion and thorax drainage. The occurrence of lung-necrosis, empyema, drained pleural fluid per body area, need of fibroscopy and Video assisted thoracoscopy (VATS), total length of stay in PICU and in hospital, days of oxygenothepy needed and the evolution of CRP were compared in the three groups. No differences on the occurrence of empyema, the need of VATS, length of hospital stay in hospital and in PICU stay, the number of oxygenothepy days and the CRP evolution. But there were less occurrence of lung-necrosis, more drained pleural fluid and less need of fibroscopy in the IPV group.

Conclusions These preliminary results show that the different chest physiotherapy has not been effective in reducing hospital stay, length of oxygenotherapy in patients with pleural effusion and thorax drain when compared to the CG. In the IPV group less complications has occurred.

\section{HYPOALBUMINEMIA IS INDEPENDENTLY ASSOCIATED WITH MORBIDITY AND MORTALITY IN CRITICALLY ILL CHILDREN}

doi:10.1136/archdischild-2012-302724.0825

HP Leite, SB de Oliveira Iglesias, LP Lima, SV de Oliveira. Pediatrics, Federal University of São Paulo, São Paulo, Brazil 